\title{
Exemplification in academic discourse structure
}

\author{
Ewa Kucelman \\ Pedagogical University of Cracow, Poland
}

\begin{abstract}
The study examines the role that exemplification plays in academic discourse. As the latest approaches emphasize, discourse is an interactional activity involving as participants both the writer and the reader. In order to ensure the proper understanding of his/her message, writers make use of different discourse strategies such as reformulation, specification, generalization or elaboration. We focus on how exemplification, viewed as the satellite, contributes to the better recognition of the subject matter, which is understood as the nucleus. In the first two sections of the study, we present an overview of discourse relations which call for the use of constructions applied in exemplification. The second part, which is based on the linguistic material obtained from a close scrutiny of two classic articles from the field of linguistics and one linguistic textbook, is devoted to the description of how exemplification contributes to specification and elaboration. We try to find and describe the specific relations, for example set-member, whole-part, process-step and object-attribute which hold between the nucleus and the satellite. Finally, we attempt at listing discourse areas which call for exemplification. The study illustrates that what are known as separate discourse relations are in fact closely related.
\end{abstract}

Keywords: exemplification, discourse structure, discourse relations, academic discourse

\section{Introduction}

The present study seeks to describe the role that exemplification plays in discourse structure, in particular, in the structure of academic discourse. It aims to pinpoint the place of exemplification in the elaborate network of discourse relations and to examine the close relationship of elaboration and explanation with respect to exemplification.

Exemplification plays a significant role in discourse structure. In the most general sense, it contributes to the creation of a coherent text by providing an easily accessible link between a more abstract, general statement of high complexity and its particular, specific instances. It positively affects coherence through enhancing the audience's identification with the message conveyed, by making discourse easier to follow and more readily acceptable.

Exemplification is a relation central to all kinds of discourse, yet in academic discourse its role is even more prominent owing to a number of characteristic features of this particular type of text. One of the most conspicuous ones is directly related to the purpose of academic 
discourse. Even more than anywhere else, the writer's aim is to be not only understood but also accepted. Writers inevitably deal with matters of high complexity, which call for the use of specific terminology, if such terminology is already available. If not, new terminology has to be coined to describe processes or phenomena so far unnamed. Thus, to ensure proper understanding of the discourse, the writer must predict where certain repairing techniques, typically associated with elaboration, will be necessary.

Hyland (2007: 268) refers to conscious attempts of the writer at producing a highly comprehensible and maximally convincing text as "metadiscourse," which he defines as follows: metadiscourse is "self-reflective matter which makes reference to the evolving text or to the writer and the imagined reader of that text." To structure the text properly, the writer has to possess thorough knowledge of a particular discourse community. The differences in the imagined target audiences are clearly visible while comparing scientific articles, targeting fellow scholar audience and pedagogical publications, which are addressed to students with limited knowledge and abilities, as will be illustrated later in the paper. Further, as Hyland claims, message processing is facilitated by code-glosses, i.e. items which supply additional information through paraphrase, explanation or elaboration. Exemplification is closely associated with the latter two.

The present study involves the examination of exemplification as used in three wellknown influential publications from the field of linguistics: Ronald W. Langacker's (2006) "Introduction to Concept, Image, and Symbol," Tanya Reinhart and Eric Reuland's (1993) "Reflexivity" and Andrew Carnie's (2006) "Syntax: a generative introduction. Second Edition." While the first two publications could be labelled as academic articles, the last one is a textbook with an explicitly acknowledged pedagogical nature. The analysis is carried out within the framework outlined in William C. Mann and Sandra Thompson's (1988) "Rhetorical Structure Theory: Toward a functional theory of text organization," where exemplification is viewed as a technique used to represent different elaboration discourse relations.

The paper is divided into three main sections. The first one defines elaboration and presents the most important elaboration relations. The second section focuses on explanation, another type of relation which calls for the application of exemplification. The core of the paper constitutes the third section, where exemplification relations are defined, ways or exemplification marking are presented and types of exemplification are identified.

\section{Elaboration relations}

Since exemplification plays the most prominent role in those discourse relations which are associated with elaboration (as illustrated by (2) to (6) below, where the satellite utterance is invariably an example), it seems reasonable to spare some time to discuss them in more detail. Elaboration itself has been given various definitions, some of which are quoted below.

Hobbs (1985: 18) defines elaboration as follows: "infer the same proposition $\mathrm{P}$ from the assertions of $S_{0}$ and $S_{1}$," where $S_{0}$ and $S_{1}$ are, in fact, seen to be identical and illustrates it with the following example: 
(1) Go down First Street.

Just follow First Street three blocks to A Street.

Characteristically, the second assertion is more detailed and provides additional information crucial for the proper understanding of the proposition in question. Here, the relation of identity holds between going down First Street and going three blocks to A Street. The second assertion, however, is more specific as it provides more detailed information concerning the length of the path.

As illustrated above, similar though the two assertions appear to be, there is a major difference between them with respect to their level of generality. This important difference constitutes the core of elaboration, as described by Mann and Thompson (1988: 273):

' $\mathrm{R}$ [a reader] recognizes the situation presented in $\mathrm{S}$ [a satellite utterance] as providing additional detail for $\mathrm{N}$ [a nucleus]. $\mathrm{R}$ identifies the element of the subject matter for which detail is provided.'

The nucleus utterance is one of a higher level of generality. As such it may be perceived by the reader or hearer as communicatively unsuccessful and consequently it calls for the adoption of some kind or repairing measure in the form of a more detailed satellite utterance. This is exactly the role of the second assertion in (1) above. The first utterance outlines the correct path to follow, yet it is imprecise as to how long the path is. This shortcoming is repaired by the second utterance, which makes the distance and the landing point more specific.

Mann and Thompson (1988: 273) acknowledge the fact that elaboration is not associated with one particular type of discourse relations, and consequently list five most important elaboration relations, namely:

- $\quad$ set-member relations, i.e. relations in which the nucleus utterance refers to the whole set denoted by a given general concept and the satellite utterance names a particular, concrete member of this set, as exemplified by (2), where "appositional constructions" form the set, and "my good friend Ollie North" is one of its members.

(2) Appositional constructions involving two nominal predications, e.g. my good friend Ollie North, are straightforwardly accommodated in this framework by means of a correspondence established between the nominal profiles. (Langacker p. 56)

- $\quad$ whole-part relations, where the nucleus utterance refers to the whole denoted by a given general concept and the satellite names its more specific parts, as in (3), in which conceptualization represents the whole and particular concepts are its parts.

(3) The term conceptualization is interpreted quite broadly: it encompasses novel conceptions as well as fixed concepts; sensory, kinesthetic, and emotive experience; recognition of the immediate context (social, physical, and linguistic); and so on. (Langacker p. 30)

- $\quad$ process-step relations, which are a characteristic feature of pedagogical discourse, where the operation of a general process is divided into several steps. In (4) below, the process of drawing tree diagrams is broken into a sequence of consecutive steps. 
(4) This method for tree drawing often works best for beginners. Here are some (hopefully helpful) steps to go through when drawing trees, below illustrated in [3]:

[3] 1. Write out the sentence and identify the parts of speech:

$\begin{array}{lllllll}\mathrm{D} & \text { Adv } & \text { Adj } & \mathrm{N} & \mathrm{V} & \mathrm{D} & \mathrm{N} \\ \text { The } & \text { very } & \text { small } & \text { boy } & \text { kissed } & \text { the } & \text { platypus. }\end{array}$

2. Identify what modifies what. Remember the modification relations. If the word modifies something then it is contained in the same constituent as that thing.

Very modifies small. Very small modifies boy.

The modifies boy. The modifies platypus.

The platypus modifies kissed.... (Carnie p. 79)

- $\quad$ object-attribute relations, in which the nucleus utterance refers to a specific object, while the satellite utterance focuses on one of the attributes of this object. In (5) the object referred to is the "referentially independent $(+\mathrm{R})$ head of the chain," while the satellite describes its property (attribute),i.e. the c-commanding function.

(5) Given [80], the referentially independent $(+\mathrm{R})$ element of the chain must be its head; that is, it must c-command the referentially dependent (- R) element. (Reinhart and Reuland p. 713)

- generalization-specification relations, which in my material constitute by far the most numerous type of elaboration relations. Here a general concept, which is often open to different interpretations is made more specific and more precise by the satellite utterance, as "imagery" in (6):

(6) Equally significant for semantic structure is the "conventional imagery" inherent in the meaning of an expression. By imagery, I do not mean sensory images à la Shepard (1978) or Kosslyn (1980), though sensory images - as one type of conceptualization - are quite important for semantic analysis. I refer instead to our manifest capacity to structure or construe the content of a domain in alternate ways. (Langacker p. 33)

The abovementioned five elaboration relations may be expressed through a few elaboration types, among which the following four constitute the most numerous group: reformulation, as in (7), where "basic domains" are rendered as "cognitively irreducible representational spaces or fields of conceptual potential," specification, as in (8), where abstract X and Y heads are replaced with specific head types, i.e. Adv and Adj, generalization in (9), where the more specific suffix $-s$ is used to illustrate a more general statement concerning the homophonous nature of English inflectional morphemes and summary (the concluding statement) in (10) below:

(7) It is however necessary to posit a number of "basic domains", i.e. cognitively irreducible representational spaces or fields of conceptual potential. (Langacker p. 32)

(8) If one phrase, XP (AdvP) modifies some head Y (Adj), then the XP must be a sister to Y (i.e., the AdvP must be a sister to the head Adj), meaning they must share a mother. You'll notice that this relationship is asymmetric: AdvP modifies Adj, but Adj does not modify AdvP. (Carnie p. 69)

(9) In many cases, the same affix can be used in very different ways. For example, the inflectional suffix -s is found both as a marker of present tense in the third person, he walks and as the plural marker, peanuts. In 
fact, leaving aside the difference in punctuation (the apostrophe), it is also used to mark possessors: John's backpack; its cover. A similar effect is seen with many other suffixes. For example -er is used both derivationally to form nouns: dancer, and as a comparative inflectional marker on adjectives: bigger. Because so many suffixes in English are homophonous (sound the same, but have different usages), be very careful when using them for morphological distribution tests. (Carnie p. 39)

(10) It is claimed instead that semantic structures (which I call "predications") are characterized relative to "cognitive domains", where a domain can be any sort of conceptualization: a perceptual experience, a concept, a conceptual complex, an elaborate knowledge system, etc. [...]Consider some examples. [...]Pushing things to their logical conclusion, we must recognize that linguistic semantics is not an autonomous enterprise, and that a complete analysis of meaning is tantamount to a complete account of developmental cognition. (Langacker p. 32)

For Corston Oliver (1998: 81) the most important aspect of elaboration relations is subject continuity described as: "the most important kind of referential continuity for identifying discourse relations." Among others, this continuity can be signaled through the application of various types of coreferential elements. In (1), for example, such is the role of the repetition of "First Street". In this way, elaboration is to be seen as closely related to various types of anaphoric relations (cf. also Bärenfänger et al. 2008).

\section{Explanation}

Apart from elaboration, which very often calls for the application of exemplification to express various discourse relations related with it, exemplification in academic discourse is closely tied with explanation, albeit in a different manner. Jasinskaja and Karagjosova (2011) define explanation as a set of relations where the second utterance gives support to the first one by providing evidence, justification, motivation or causal explanation. Unlike elaboration, where the second utterance clarifies the meaning of the first one, explanation does not seek to support the nucleus utterance with a satellite one which asserts basically the same proposition, expressing it in a way that is easier for the intended or actual reader to identify and accept. Here, the second utterance supports the truth value of the first one. Consider, for example, utterances such as those in (11)-(13):

(11) Another change we must assume in the standard chain theory is prohibiting (80) from applying to singlemember chains. This change is needed in order to allow for the logophoric use of anaphors. (Reinhart and Reuland p. 702)

(12) The second is the domain allowing SE anaphors to be bound (though not excluding pronouns). Despite the apparently massive differences reported among languages, we argue there that this too is reducible to a unique domain. In traditional terms, this binding obeys the Tensed-S Constraint; that is, it is impossible across tense. We show that this follows from the fact that SE anaphors must move to I to acquire $\varphi$ features. (Reinhart and Reuland p. 660)

(13) The precise configuration of such a network is less important than recognizing the inadequacy of any reductionist description of lexical meaning. A speaker's knowledge of the conventional value of a lexical item cannot in general be reduced to a single structure, such as the prototype or the highest-level schema. For one thing, not every lexical category has a single, clearly determined prototype, nor can we invariably 
assume a high level schema fully compatible with the specifications of every node in the network (none is shown in Figure 1). (Langacker p. 31)

where function of the utterance following the initially made statement clearly is to justify the claim made. The claim itself is not seen as obscure or difficult to comprehend; the reader will not fail to understand it, yet they may be uncertain why it needs to be made. In (11) the second sentence provides justification for the restriction on the application of a specific rule. The last sentence in (12) represents causal explanation. The requirement for SE anaphors to obtain $\varphi$-features is the cause for the Tensed-S Constraint. In (13) the main explanation relation is that of motivation. Langacker makes here a potentially controversial statement going against one of the "popular" theories. This claim is felt to require proper motivation.

Although elaboration and explanation are principally different phenomena, they are often discussed together, on the grounds that they play a very similar role in discourse structure. This is due to the fact that both in explanation and elaboration the first utterance fails to achieve the intended communicative goal and the second one has a repairing role. Both anticipate a misunderstanding or/and communication failure, which as Lascarides and Asher (2009) state most often results from problems with reference resolution (i.e. What does this refer to?) and lexical access (What does this mean?). Because of its repairing role exemplification is often associated with elaboration and explanation.

\section{Exemplification}

Hobbs (1985:20) gives the following definition of exemplification: "Infer $\mathrm{p}(\mathrm{A})$ from assertion $S_{0}$ and $p(a)$ from the assertion of $S_{1}$ where a is a member of subset of A." and supports it with the following example:

(14) This algorithm reverses a list.

If its input is "A B C", its output is "C B A".

In (14) the statement made by the first utterance is supported by the second one, giving a reversed list. Hyland (2007: 270) expresses the role that exemplification plays in discourse structure in a more straightforward manner, as "a communication process through which meaning is clarified or supported by a second unit which illustrates the first by citing an example." The characteristic feature of exemplification is that it is not necessarily associated with two formally separate utterances, as apart from functioning on a clausal level, it is typically associated with phrasal or even lexical levels.

\subsection{Exemplification marking}

In their article, Jasinskaja and Karagjosova (2011) make a strong claim, supporting it with the data from the corpus study of Taboada (2006), that elaborations are often unmarked. On the other hand, exemplifications are typically (lexically) marked off. Hyland (2007: 278) lists the following most frequently used lexical markers of exemplification: such as, for example, e.g., an example of, like, for instance and say. This does not mean that there are no lexically 
unmarked exemplifications found, as examples (15) and possibly (8), requoted here as (16) show:

(15) A predication's scope is not always sharply delimited or explicitly indicated, but the construct is nonetheless of considerable structural significance [...]. Consider the notion island with respect to the various scopes indicated in Figure 5. (Langacker p. 36)

(16) If one phrase, XP (AdvP) modifies some head Y (Adj), then the XP must be a sister to Y (i.e., the AdvP must be a sister to the head Adj), meaning they must share a mother. You'll notice that this relationship is asymmetric: AdvP modifies Adj, but Adj does not modify AdvP. (Carnie p. 69)

However, the unmarked character of the above exemplifications is far from being unquestionable. For example, Hyland (2007) does not consider exemplifications such as those in (16) unmarked. Among possible exemplification marking devices he lists punctuation, and he finds bracketing among the most frequent types of marking used. Consequently, the bracketed expressions will be infallibly identified as examples. It is also worth noting that in (15) the second utterance names the example but it doesn't state it. Therefore, we might dare to claim that the whole clause itself serves as exemplification marking here. Similar cases of finite and nonfinite clauses marking off exemplification are given in (17) and (18):

(17) Most predications also require more than one domain for their full description, in which case I refer to the set as a "complex matrix", as illustrated for knife in Figure 2. (Langacker p. 32)

(18) The base of a predication is its domain (or each domain in a complex matrix). Its profile is a substructure elevated to a special level of prominence within the base, namely that substructure which the expression "designates". Some examples are sketched in Figure 3, with the profile given in heavy lines. (Langacker p. 34)

In (17) exemplification is marked by the nonfinite clause "as illustrated" and in (18) a similar role is played by the finite clause "some examples are sketched in Figure 3."

By the same token, exemplification may be marked by prepositional phrases similar to "as in (1)" in (19):

(19) In accordance with Postal (1970), Vergnaud (1987), and others, we will assume that pronouns are in determiner position. Yet they project as full NPs, as in (1). (Reinhart and Reuland p. 658)

When exemplification is lexically marked, it is usually by employing a linking adverbial or an abbreviation corresponding to it. Typical adverbials used in exemplifications include: for example, for instance, like, such as, etc. Some examples are given in (20)-(23) below:

(20) Grammatical constructions have the effect of imposing a particular profile on their composite semantic value. When a head combines with a modifier, for example, it is the profile of the head that prevails at the composite structure level. (Langacker p. 41)

(21) The nodes and categorizing relationships in such a network differ in their degree of entrenchment and cognitive salience - for instance, the heavy-line box in Figure 1 corresponds to the category prototype. (Langacker p. 31) 
(22) Similarly we can distinguish among the various kinds of determiner using features like [ $\pm w h]$, [ \pm quantifier], [ \pm deictic], etc. (Carnie p. 46)

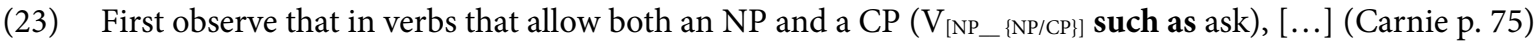

The use of abbreviations in exemplification marking is given in (24):

(24) Appositional constructions involving two nominal predications, e.g. my good friend Ollie North, are straightforwardly accommodated in this framework by means of a correspondence established between the nominal profiles. (Langacker p. 56)

\subsection{The relationship between generalization and exemplification}

The characteristic feature of many of the above examples is that they are merely introductions to exemplifications proper or comments on the previously given examples:

(25) Dutch SELF anaphors are allowed in essentially the same environments, as illustrated with the translations of $[22 a-b]$ in $[22 c-d]$.

[22] a. There were five tourists in the room apart from myself.

b. ${ }^{\star}$ Five tourists talked to myself in the room.

c. Er waren vijf toeristen in de kamer behalve mezelf.

d. ${ }^{*}$ Vijf toeristen praatten met mezelf in de kamer.

[23] a. Physicists like yourself are a godsend. (Ross 1970)

b. ${ }^{*}$ A famous physicist has just looked for yourself.

[24] a. 'She gave both Brenda and myself a dirty look.'

b. ${ }^{*}$ She gave myself a dirty look.

[...] Normally, first person reflexives behave strictly like anaphors. For example, in the (b) cases of [22][24] they are ruled out, correctly, by Condition A. Why, then, does Condition A fail precisely in the (a) cases? Furthermore, the problem is not restricted to first person reflexives. As was noted by the same scholars in the seventies, third person reflexives can be long-distance bound in English, in violation of Condition A. This use (knownas logophoric), which is assumed to interact with point of view, is most common in narrative texts [...] (Reinhart and Reuland p. 669)

The above quote from Reinhart and Reuland is in large part a list of examples of reflexives used correctly or incorrectly in English and Dutch. These examples on the one hand do exemplify the initial claim concerning the similarity between English and Dutch selfpronouns. On the other hand, however, they serve as an introduction to the generalizations made below them. Only there does the reader learn that the examples given in (a) and (b) exemplify two distant applications of reflexives, where the logophoric one is a new generalization not mentioned anywhere earlier in the discourse.

This observation naturally triggers the question concerning the relationship between the generalization and the example that exemplifies it. In most of the literature dealing with exemplification the implied assumption is that the example should linearly follow the generalization. Such a placement quite logically follows from the fact that exemplification is a relation frequently associated with elaboration and explanation, which are defined through their repairing function in discourse which is likely to be a communicative failure. Even Hyland (2007), who focuses on the role of exemplification in academic discourse, quotes only 
such cases where the exemplification follows the general statement. Yet, our material shows that the reverse ordering is not infrequent.

For the sake of simplicity, let us limit our investigation to those exemplifications which are associated with elaboration and explanation. The problem with the classical definitions of elaboration and explanation quoted in the initial sections of the present paper is that they wrongly presuppose that the nucleus utterance must precede the satellite one. This presupposition is clearly visible in Mann and Thompson's (1988: 273) list of elaboration relations, i.e. whole-part, set-member, process-step etc. In real academic discourse, however, argumentation is built in two directions, which we will refer to, adopting terminology used in chemistry, as analytic and synthetic. In analytic argumentation the nucleus utterance precedes the satellite one. It is most typically seen as a statement of some objective or at least widely acknowledged fact often already known or described, while in synthetic argumentation the writer starts with empirical data, whose interpretation leads to the development or presentation of a relevant generalization.

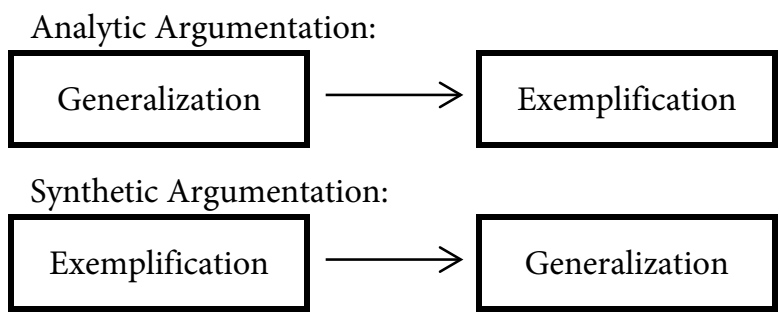

Figure 1: Analytic and Synthetic Argumentation

In (25) above, the parallel between English and Dutch reflexives is presented as a fact which is evidenced by the examples that follow. Yet, at the same a careful reader will notice that there is something bothering about examples (a) and (c); namely, they are grammatical while the theory developed so far would predict them unacceptable. This calls for synthetic argumentation, i.e. relating the "strange" cases to the general notion of logophoricity.

The choice of argumentation type in (25) is dictated by stylistic, text internal properties. In other words, the set of presented examples exemplifies two distinct generalizations. Giving them both before the examples would most likely result in the lack of communication transparency, with the reader confused as to what aspects of the examples to focus on. Separating the two generalizations seems communicatively more effective. We call such a procedure generalization - exemplification chain, which can be schematically represented as follows:

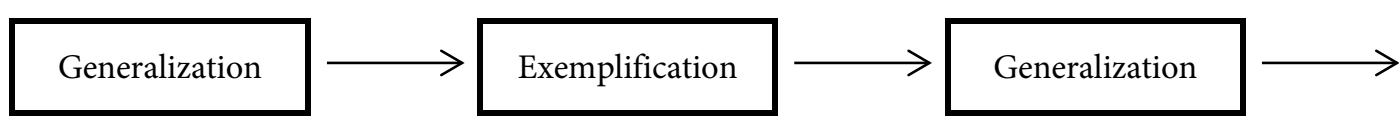

Figure 2: Generalization - elaboration chain

in other cases, the choice between analytic and synthetic argumentation is a matter of subjective preference. Synthetic argumentation is very often used in pedagogical discourse, as illustrated by (26) below, where the example of "bigger units" precedes the generalization concerning the nature of a constituent: 
(26) We have two different ways to represent this bigger unit. One of them is to put square brackets around units:

3) [the student]

The other is to represent the units with a group of lines called a tree structure:

4)

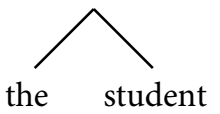

These bigger units are called constituents. An informal definition for a constituent is given in (5):

5) Constituent: A group of words that functions together as a unit.

In such types of discourse starting with a detail may be more communicatively effective than choosing an abstract generalization as a starting point.

\subsection{Exemplification types}

Exemplifications primarily have an illustrating function, making a general statement more understandable by referring to concrete, specific instances of this generalization. They explain or elaborate on the previous (or the following) predication by giving an example. Yet, there is an obvious difference between exemplifications used in elaboration and those in explanation. Secondly, while explanation exemplifications in the material analysed in this study are limited to those giving evidence, in elaboration exemplification is used in a variety of relations. Most typically we deal with two types of relations: generalization-specification and set-member. Additionally in academic texts, illustrating examples are used for process-step specification. The application of exemplifications may be viewed as governed by the following set of rules, presented below:

Generalization-Specification Rule for Exemplifications:

Make a general statement A more specific by picking one member out of the set of specific cases $\left\{a_{1} \ldots a_{n}\right\}$ which the generalization extends over.

The Generalization-Specification rule can be illustrated with the following examples:

(27) Most predications also require more than one domain for their full description, in which case I refer to the set as a "complex matrix", as illustrated for knife in Figure 2.

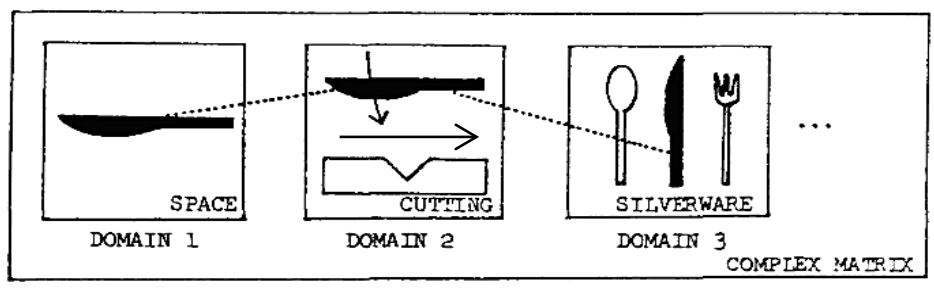

(Langacker p.32)

(28) Next, we'll do a derivation of a sentence where the wh-phrase moves from an embedded clause to the specifier of a main clause $\mathrm{CP}$.

[26] Who(m) do you think Jim kissed? (Carnie p. 325)

(29) With respect to the former, a unit is minimal (a "morpheme") if it contains no other symbolic units as components. For instance, despite its internal complexity at both the semantic and the phonological poles, 
the morpheme sharp is minimal from the symbolic standpoint, whereas sharpen, sharpener, and pencil sharpener are progressively more complex. (Langacker p. 45)

In (27), the general term "complex matrix" has been illustrated with an example of such a matrix that can be drawn for a specific lexical entry, i.e. "knife". What is worth noting is that exemplification is not necessarily limited to linguistic representations. Here the example proper is a schematic figure. It is a fact that academic discourse is highly dependent on nonlinguistic devices, such as figures, graphs, charts, drawings etc., many of which serve the exemplification function. This naturally opens a discussion which is beyond the scope of the present paper as to the relationship between the linguistic and non-linguistic elements in discourse.

In (28) the derivation of a sentence with a wh-movement to the specifier position of the matrix CP is presented using the example of a clause in which such a movement takes place. In (29) the concept of a minimal unit is exemplified with the morpheme "sharp" and its more composite, complex forms "sharpen" and "sharpener".

Set-member exemplifications are subject to the following rule:

Set-Member Rule for Exemplifications:

Make the set generally defined by utterance A more accessible by picking out one of its members $\left\{p_{1} \ldots p_{n}\right\}$.

The operation of this rule is visible in (30), (31) and (32).

(30) In spoken varieties of English (both standard and non-standard), function words often contract with nearby words. One such contraction takes non-finite $\mathrm{T}$ (to) and contracts it with a preceding verb like want:

i) I want to leave $\rightarrow$ I wanna leave. (Carnie p. 322)

(31) Consider the argument based on verb/noun pairs which refer to the same process, e.g. extract and extraction. (Langacker p. 49)

(32) Appositional constructions involving two nominal predications, e.g. my good friend Ollie North, are straightforwardly accommodated in this framework by means of a correspondence established between the nominal profiles. (Langacker p. 56)

In example (30), the set described is one composed of function words combining with nearby words to form a contraction. Out of this set, as an example, a specific subset is extracted, i.e. the set formed by a lexical verb followed by the infinitive marker 'to'. Subsequently, from the extracted subset a particular member is chosen as a representative, in our case, the verb 'want.' Thus, it may be observed that the Set-Member Rule can operate in two stages. It is the case when the set in question is not a homogenous one. In English, typical contractions involve also the negative marker 'not' contracting with the preceding auxiliary, and auxiliaries contracting with a preceding NP. Consequently, it seems desirable that the reader be informed that the example represents only a particular type of the construction rather than the set as a whole. In (31) and (32), the Rule operates in one stage only, as the sets formed by derivationally related pairs of verbs and nouns and appositional nominal constructions are believed to be homogenous ones. 
The last group, namely process-step exemplifications are in our material found chiefly in pedagogical academic discourse, probably because certain procedures are anticipated by the writer to be potentially unclear and difficult to understand by the inexperienced student reader. What seems interesting to note is that the examples themselves do not form the process-step relation. Instead, they perform an illustrating function to each step of the process, as in (33) and (34):

(33) This method for tree drawing often works best for beginners. Here are some (hopefully helpful) steps to go through when drawing trees.

1. Write out the sentence and identify the parts of speech:

$\begin{array}{lllllll}\mathrm{D} & \text { Adv } & \text { Adj } & \mathrm{N} & \mathrm{V} & \mathrm{D} & \mathrm{N} \\ \text { The } & \text { very } & \text { small } & \text { boy } & \text { kissed } & \text { the } & \text { platypus. }\end{array}$

2. Identify what modifies what. Remember the modification relations. If the word modifies something then it is contained in the same constituent as that thing.

Very modifies small.

Very small modifies boy.

The modifies boy.

The modifies platypus.

The platypus modifies kissed.

[...] (Carnie p. 79)

(34) 1. This method starts out the same way as the other: write out the sentence and identify the parts of speech.

$\begin{array}{lllllll}\mathrm{D} & \text { Adv } & \text { Adj } & \mathrm{N} & \mathrm{V} & \mathrm{D} & \mathrm{N} \\ \text { The } & \text { very } & \text { small } & \text { boy } & \text { kissed } & \text { the } & \text { platypus. }\end{array}$

2. Next draw the TP node at the top of the tree, with the subject NP and VPunderneath:

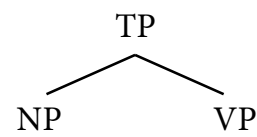
D Adv Adj $\mathrm{N} \quad \mathrm{V} \quad \mathrm{D} \quad \mathrm{N}$
The very small boy kissed the platypus.

3. Using the NP rule, flesh out the subject NP. You will have to look ahead here. If there is a P, you will probably need a PP. Similarly, if there is an Adj, you'll need at least one AdjP, maybe more. Remember the principle of modification: elements that modify one another are part of the same constituent.

[...] (Carnie p. 82)

Consequently, the rule governing the application of exemplification in process-step relations may be worded as follows:

Process - Step Rule for Exemplifications:

Use exemplification to illustrate each step of the process if in the process-step relation the utterance referring to the process is not made accessible enough through listing its consecutive steps.

Whole-part relations do not represent typical exemplifications. In (2), requoted here as (35) we deal, in fact, with listing rather than exemplification, although 'and so on' at the end of the list may apparently suggest that the enumerated conceptions are examples with a typical illustrating function. At the same time, it must be remembered that it is often impossible to present an exhaustive list of the component parts, both because it might be infinitely long and simply because it is not really necessary or desirable to name all the parts. I am in favour of keeping listings and exemplifications apart. 
(35) The term conceptualization is interpreted quite broadly: it encompasses novel conceptions as well as fixed concepts; sensory, kinesthetic, and emotive experience; recognition of the immediate context (social, physical, and linguistic); and so on. (Langacker p. 30)

In my indeed very limited material, exemplification is predominantly related to elaboration. However, in academic discourse we also find extended chains where a certain general utterance in illustrated with an example, which in turn calls for additional explanation.

(36) [12]

Condition B

A reflexive predicate is reflexive-marked.

Let us now look at how this condition works in the examples we have been discussing. In the case of [6a] (repeated in [13]), [12] is equivalent to the standard Condition B: in both [13a] and [13b] binding yields a reflexive predicate, so Condition $B$ requires reflexive marking. Whereas [13b] is appropriately marked, the unmarked [13a] is filtered out. We may note that Condition B also captures cases left for Condition C in the standard binding theory. For [12], there is no difference between [13a] and [13c], and binding is ruled out in both, for the same reason.

[13]

a. ${ }^{*} \operatorname{Max}_{1}$ criticized him 1 .

b. Max ${ }_{1}$ criticized himself 1 .

c. ${ }^{*} \operatorname{Max}_{1} /$ he 1 criticized $\operatorname{Max}_{1}$ (Reinhart and Reuland p. 663)

In (36) the examples in [13] are meant to illustrate the operation of Condition B, as presented in [12]. Yet, the examples themselves are not seen as accessible enough and writers predict a possible communication failure. Consequently, they reach for another repairing method, namely explanation. Using exemplification as a form of elaboration and then explanation to make the examples more informative constitutes another characteristic feature of academic discourse, where elaboration - exemplification-explanation chains are the order of the day. Such chains are presented schematically in Figure 3 below:

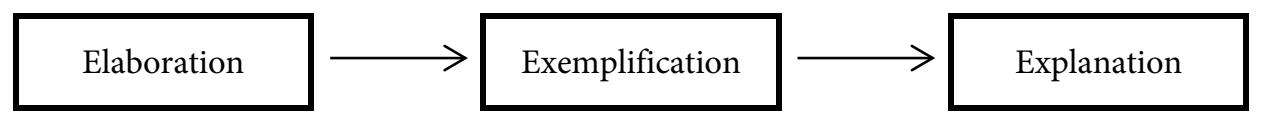

Figure 3: Elaboration - exemplification - explanation chain

Another illustration of the above chain, this time with a schematic, partly non-linguistic exemplification followed by explanation is given in (37):

(37) The base of a predication is its domain (or each domain in a complex matrix). Its profile is a substructure elevated to a special level of prominence within the base, namely that substructure which the expression "designates". Some examples are sketched in Figure 3, with the profile given in heavy lines. The base (or domain) for the characterization of hypotenuse is the conception of a right triangle; for tip, the base is the conception of an elongated object; and for uncle, a set of individuals linked by kinship relations. The base is obviously essential to the semantic value of each predication, but it does not per se constitute that value: a hypotenuse is not a right triangle, a tip is not an elongated object, and an uncle is not a kinship network. 

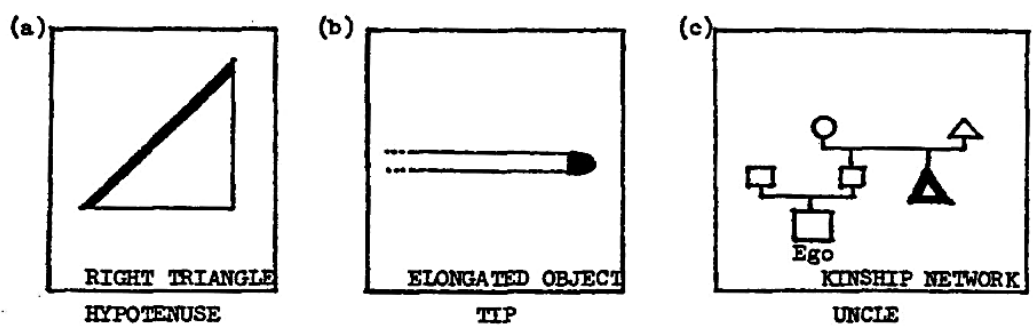

(Langacker p.34)

\section{Conclusions}

Exemplification, as a tool used in elaboration, is a conscious measure taken by the writer to avoid communicative failure. It makes it possible to make a general concept more accessible by referring to a specific case, or by pointing to specific members of a particular set. Exemplification may also play a supplementary role in process-step relations, where particular steps are illustrated with specific examples. However, exemplification itself may sometimes fall short of the target. In such a case explanations are necessary.

Exemplification is sometimes involved in relations that go beyond the standard twoelement relations. The two most characteristic chains exemplification is part of are generalization-exemplification-generalization and elaboration-exemplification-explanation chains. The network of exemplification relations is presented schematically in Figure 4 below:

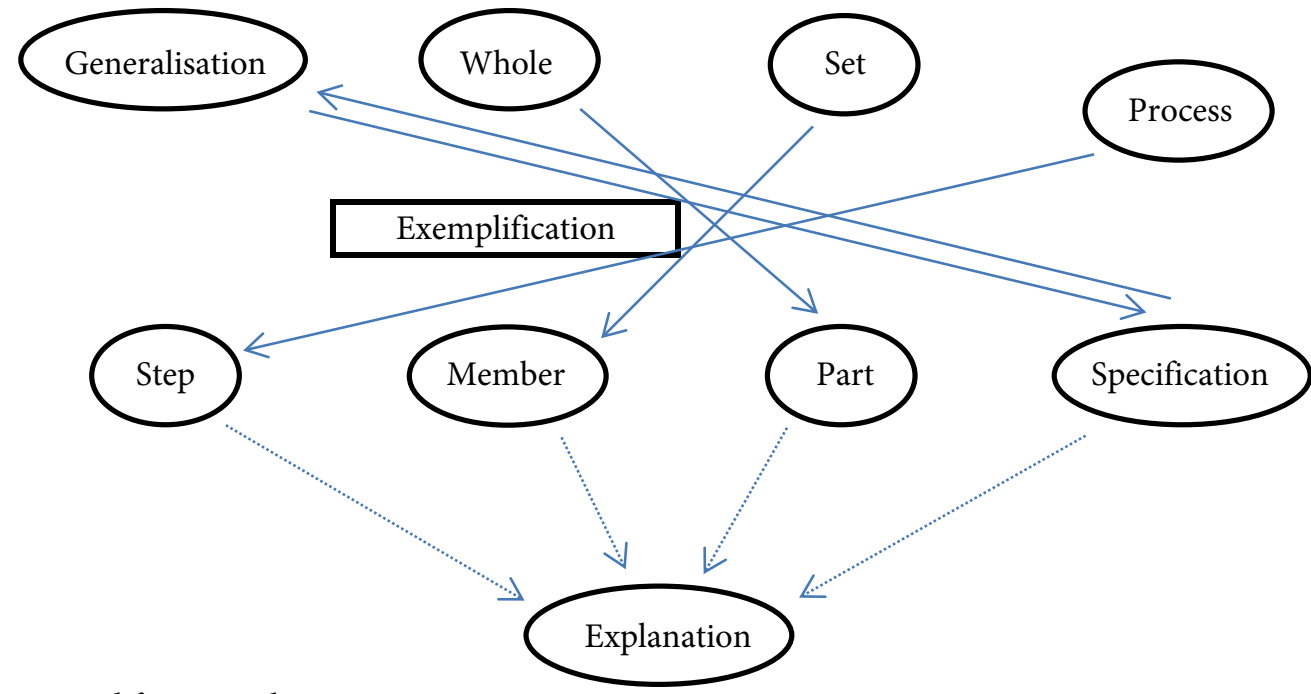

Figure 4: Exemplification relations

In Figure 4, the arrows show the direction in which the text develops, that is, for example, from a statement describing the whole set to the statement referring to its particular member or members. The arrows show the typical direction in which the argumentation develops, i.e. from the process to step rather than the other way round. With generalization-specification relation, where argumentation can develop in both directions, two arrows are used. All the lines pass through the exemplification box. Exemplification should be interpreted as a means of achieving the target. Thus, e.g. in the process - step relation, the consecutive steps or the 
process are illustrated with specific examples. Finally, the dotted lines that join the relations with explanation represent the possibility of building chains o relations.

\section{Primary Sources:}

Langacker, R.W. 2006. Introduction to Concept, Image, and Symbol. In D. Geeraerts (ed.), Cognitive Linguistics: Basic Readings, 29-68. Berlin: Mouton de Gruyter.

Reinhart, T., and E. Reuland. 1993. Reflexivity. Linguistic Inquiry 24: 657-720.

Carnie, A. 2006. Syntax: a generative introduction. Second Edition. Malden: Blackwell Publishing.

\section{References}

Asher, N., and L. Vieu. 2005. Subordinating and coordinating discourse relations. Lingua 115: 591-610.

Bärenfänger, M., D. Goecke, M. Hilbert, H. Lüngen, and M. Stührenberg. 2008. Anaphora as an indicator of elaboration: A corpus study. JLCL 23/2: 49-73.

Corston-Oliver, S.H. 1998. Computing of Representations of the Structure of Written Discourse. PhD thesis, University of California, Santa Barbara.

Cote, S. 1997. Elaboration: A function and a form. Proceedings of the 23rd Annual Meeting of the Berkeley Linguistic Society.

Danlos, Laurence. 2001. Event coreference in causal discourses. In P. Bouillon, and F. Busa (eds.), The Language of Word Meaning, 216-241. Cambridge: Cambridge University Press.

Halliday, M.A.K., and R. Hasan. 1976. Cohesion in English. London: Longman.

Hobbs, J.R. 1985. On the coherence and structure of discourse. CSLI.

Hyland, K. 2007. Applying a gloss: exemplification and reformulation in academic discourse. Applied Linguistics 28(2): 266-285.

Jasinskaja, K., and E. Karagjosova. 2011. Elaboration and Explanation. In Proceedings of Constraints in Discourse IV, September 14th-16th, 2011, Agay-Roches Rouges, Var, France.

Knott, A., J. Oberlander, M. O'Donnell, and C. Melish. Beyond elaboration: the interaction of relations and focus in coherent text. In T. Sanders, J. Schilperoord, and W. Spooren (eds.), Text Representation: Linguistic and Psycholinguistic Aspects, 181-196. Amsterdam/Philadelphia: Benjamins.

Lascarides, A., and N. Asher. 2009. Agreement, disputes and commitments in dialogue. Journal of Semantics 26(2): 109-158.

Mann, W.C., and S. Thompson. 1987. Rhetorical Structure Theory: Description and Construction of text Structures. In G. Kempen (ed.), Natural Language Generation: New Results in Artificial Intelligence, Psychology and Linguistics, 85-95. Boston: Martinus Nijhoff Publishers.

Mann, W.C., and S. Thompson. 1988. Rhetorical Structure Theory: Toward a functional theory of text organization. Text 8(3): 243-281.

Polanyi, L. 1988. A formal model of the structure of discourse. Journal of Pragmatics 12: 601-638.

Taboada, M. 2006. Discourse markers as signals (or not) of rhetorical relations. Journal of Pragmatics 38: 567-592.

Taboada, M., and W.C. Mann. 2006. Rhetorical Structure Theory: looking back and moving ahead. Discourse Studies 8(3): 423-459.

Webber, B., M. Egg, and V. Kordoni. 2012. Discourse structure and language technology. Natural Language Engineering 18: 437-490. 